\title{
High Order Thinking Skills and Their Effect on Knowledge Accumulation
}

\author{
Sylvia Encheva ${ }^{1 *}$ \\ ${ }^{1}$ Stord/Haugesund University College, Bjørnsonsg. 45, 5528 Haugesund, Norway \\ ${ }^{*}$ Corresponding author
}

\begin{abstract}
Knowledge and skills students gather for a period of time is difficult to estimate. Learning is effected by a number of factors and prior knowledge is among the very important ones. Despite existence of a substantial amount of tests for assessing newly obtained knowledge and skills, there is still a need to provide tailored responses to answers students submit by connecting those answers to their prior knowledge. Lattice structures prove themselves to be especially useful for unveiling dependences among various factors.
\end{abstract}

Keywords-logic; learning; graphs

\section{INTRODUCTION}

Establishing amount of knowledge and skills obtained by students during a semester is a very complicated task. The process gets even harder when preliminary knowledge play a crucial role for learning new concepts which in addition should be internalized in a very short period of time. It is well known that in some subjects like for example mathematics insufficiency of certain knowledge and skills are a real hindrance for further advancement.

Various approaches for data mining and knowledge discovery are presented in [12]. An information theoretic approach for testing students knowledge bases is presented in [3]. Evaluation and interpretation of knowledge is discussed in [11]. Knowledge evaluation of implicit learning is in the focus of the work described in [8].

Importance of higher order thinking skills is pointed in [6], while different ways to assess higher order thinking skills are presented in [2]. A number of approaches to increase higher order thinking can be found in [13].

Below we propose an approach for providing individual feedback to students regarding their performance during a particular study and extracting information about some groups' tendencies. Such structured experience can become quite handy when the same course is given to new students. While individual development is often related to personal life situations group performance can indicate a need for changes in teaching settings. For assessing students' higher order thinking skills we propose application of default lattices.

The rest of the paper goes as follows. Theoretical supporting the study is presented in Section 2, our approach can be found in Section 3 while the conclusion is placed in Section 4.

\section{PRELIMINARIES}

A lattice is a partially ordered set, closed under least upper and greatest lower bounds. The least upper bound of $X$ and y is called the join of $x$ and $y$, and is sometimes written as $x+y$; the greatest lower bound is called the meet, [5], [7], and [9]. A partially ordered set in which all pairs have a meet is meet-semilattice, [7].

A bilattice is a set equipped with two partial orderings truth ordering $\leq_{t}$ and knowledge ordering $\leq_{k}$. The $t$ partial ordering $\leq_{t}$ means that if two truth values $a, b$ are related as $a \leq_{t} b$ then $b$ is at least as true $a$. The $k$ partial ordering $\leq_{k}$ means that if two truth values $a, b$ are related as $a \leq_{k} b$ then $b$ labels a sentence about which we have more knowledge than a sentence labeled with $a$.

Bilattices put together two lattice orders one measuring 'degree of truth', and another one measuring 'degree of knowledge', [10]. Knowledge in a bilattice is usually measured vertically while the truth is measured horizontally. Default consequences are results of two modes of inferences skeptical or credulous reasoning. The logic that can be used for default reasoning has the following truth values: $t$ - true, $f$ false, $\perp$ - undefined, $\mathrm{T}$ - contradictory, $d \mathrm{~T}$ - contradictory by default, $d t s$ - skeptically true by default, $d f s$ - skeptically false by default, $d t c$ - credulously true by default, $d f c$ credulously false by default, and * - undetermined by default.

\section{TeSt OUtCOMES AND PREVIOUS RESUlts}

Students' abilities to analyze, evaluate, and create (as described in the revision of Bloom's taxonomy [1]) can be classified by assigning their test results to nodes in a bilattice Fig. 1 as follows.

Two correct answers imply that a student possesses high level thinking skills. The assigned truth-value is $t$. One correct answer and one partially correct answer imply doubt about the student's possession of high level thinking skills. The assigned truth-value is dts. One correct answer and one unanswered question imply doubt about the student's possession of high level thinking skills. The assigned truth-value is dtc. One correct answer and one wrong answer imply some doubt about the student's possession of high level thinking skills. The 
assigned truth-value is T. Two partially correct answers imply doubt about the student's possession of high level thinking skills. The assigned truth-value is $d \mathrm{~T}$.

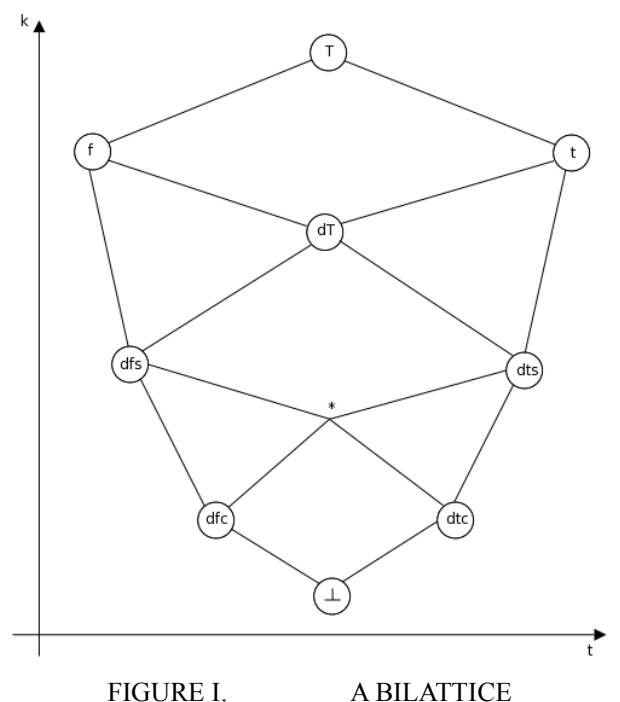

One partially correct answer and one unanswered question imply doubt about the student's possession of high level thinking skills. The assigned truth-value is *. One partially correct answer and one wrong answer imply doubt about the student's possession of high level thinking skills. The assigned truth-value is $d f s$. One wrong answer and one unanswered question imply doubt about the student's possession of high level thinking skills. The assigned truth-value is $d f_{c}$. Two wrong answers imply lack of high level thinking skills. The assigned truth-value is $f$. Two unanswered questions imply doubt about the student's possession of high level thinking skills. The assigned truth-value is $\perp$. Appropriate reading materials and tutorials are to be recommended to each student according to the assigned to her truth-value.

Apart from using bilattice structure for providing automated recommendations to students, a lecturer can look at the content of each node in a bilattice and make a connection between students past and current performances. This can indicate which misunderstandings and or misconceptions should be further addressed.

Effect of results of previously taken mathematics courses on current knowledge status is incorporated in feedback to students by introducing a new coefficient

$$
\sigma=\frac{\frac{w_{1} g_{1}}{g}+\ldots+\frac{w_{n} g_{n}}{g}}{n}=\frac{w_{1} g_{1}+\ldots+w_{n} g_{n}}{n g}
$$

where $g_{i}$ stands for the grade for exam number $i, 1 \leq i \leq n$, $w_{i}$ is the assigned weight for $g_{i}$, while $g$ stands for the max grade. In case exam grades are given in text form we suggest scaling them as in [9].

Feedback to students is based on an outcome of the following calculations

$$
\Phi=\frac{w_{\tau} \tau}{n g} \sum_{i=1}^{n} w_{i} g_{i}=w_{\tau} \cdot \tau \cdot \sigma
$$

where $w_{\tau}$ is the assigned weight for a test score $\tau$

$$
w_{\tau}+\sum_{i=1}^{n} w_{i}=1
$$

The outcomes imply which students are very likely to experience difficulties reaching sufficient level of knowledge and skills while studying this subject. Obviously the value of $\Phi$ can be calculated in a similar way when there are several partial exams during a semester and weights are to be reconsidered when a new group of students takes the same course.

Up to this point we were following individual progress developments. In order to find somewhat more general occurences we propose application of concept lattices.

In a concept lattice concepts are presented in boxes where the lower row lists concepts objects and the upper row lists concepts attributes. When two concepts are connected with a straight line one can see that the lower concept contains less objects and more attributes while the upper concepts has less objects and more attributes.

Concepts in a lattice can illustrate which groups of students have the same results related to particular topics and where they differ in their performance. Students can be placed in three groups 'high', 'average', and 'low' according to their exam grades. We can see what 'high and average' groups learn equally good and where exactly are the differences between 'high' and 'average' groups. This can support provision of tailored advise to students in group 'average' that will help them to join the 'high' group. Similarly, students from the 'low' group can receive personal advise on how to join the 'average' group and possibly the 'high' group. An example is provided in Fig. 2. Students with the same results related to particular topics can be found in concepts placed on the third and fourth rows in Fig. 2. Their differences can be seen by following superconcepts placed in the fifth and sixth rows in Fig. 2.

Concepts lattices are most of the time used to show dependences between attributes and objects in a positive way, i.e. a set of objects has a set of attributes. In case of evaluating learning one can ask a nearly 'dual' question - which attributes are not possessed by groups with low level of performance? Such information can be further incorporated in the process of providing automated help, suggesting learning objects and additional

learning 


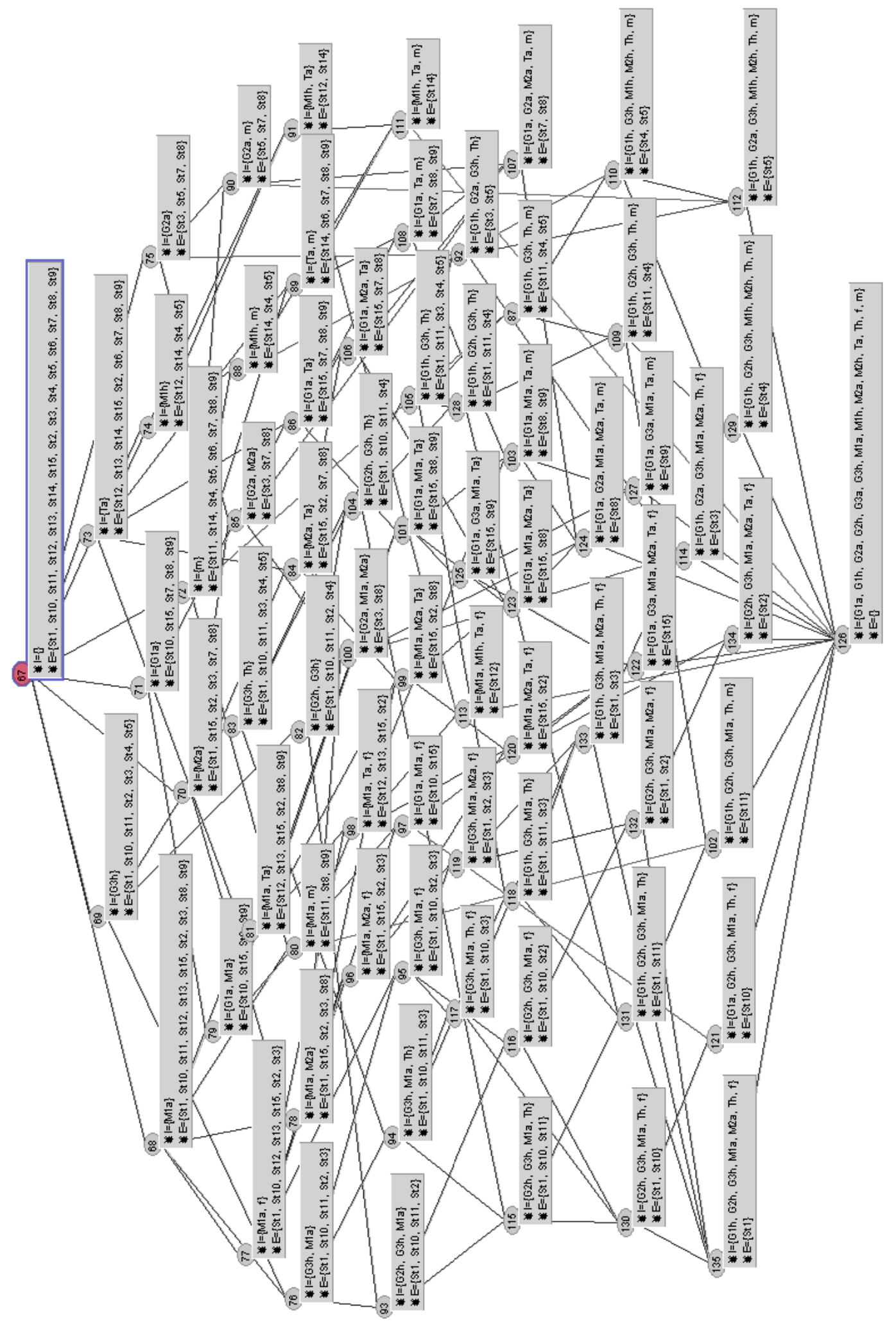

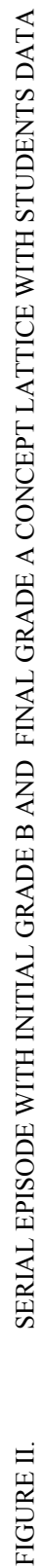




\section{CONCLUSION}

While most tests establish levels of subject related knowledge it is also very important to work on developing tests establishing levels of higher order thinking skills. A large number of students believe that factual knowledge is the only one that is missing. Educators however should work on developing students' higher order thinking skills regardless which course they are giving.

\section{REFERENCES}

[1] L. W.Anderson and D. R. Krathwohl, A Taxonomy for Learning, Teaching, and Assessing: A Revision of Bloom's Taxonomy of Educational Objectives, New York: Longman, 2001.

[2] S. M. Brookhart, How to Assess Higher-Order Thinking Skills in Your Classroom, ISBN-13: 978-1-4166-1048-9, 2010.

[3] J. E. Bruno, "Assessing the knowledge base of students: An information theoretic approach to testing," Journal of Measurement and Evaluation in Counselling and Development, vol. 19(3), pp. 116-130, 1986.

[4] L. M. Cabrer, A. P. K. Craig, and H. A. Priestley, Product representation for default bilattices: an application of natural duality theory, Journal of Pure and Applied Algebra, vol. 219, pp. 2962-2988, 2015.

[5] C. Carpineto and G. Romano, Concept Data Analysis: Theory and Applications. John Wiley and Sons, Ltd., 2004.

[6] R. Collins, "Skills for the 21st Century: teaching higher-order thinking," Independent Schools Queensland, vol. 12 (14), 2014.

[7] B. A. Davey and H. A. Priestley, Introduction to lattices and order. Cambridge University Press, Cambridge, 2005.

[8] D. Fum and A. Stocco, "Outcome Evaluation and Procedural Knowledge in Implicit Learning”, Proceedings of the 25th Annual Meeting of the Cognitive Science Society, pp. 456-431, 2003.

[9] B. Ganter and R. Wille, Formal Concept Analysis, Springer, 1999.

[10] M. L. Ginsberg, Multivalued logics: a uniform approach to inference in Artificial Intelligence, Computational Intelligence, vol. 4, pp. 265-316, 1988.

[11] J. Guan and J. Wang, Evaluation and interpretation of knowledge production efficiency, Evaluation, vol. 59(1), pp. 131-155, 2004.

[12] W. Klosgen and J. Zytkow, Handbook of data mining and knowledge discovery, Oxford University Press, Inc. New York, NY, USA, 2002.

[13] A. Thomas and G. Thorne, "How to Increase Higher Order Thinking", Metarie, LA: Center for Development and Learning, 2009. 